\title{
A Nonlinear Optimization Technique of Tunnel Construction Based on DE and LSSVM
}

\author{
Xing Jun, ${ }^{1}$ Jiang Annan, ${ }^{2}$ Wen Zhiwu, ${ }^{2}$ and Qiu Jingping ${ }^{1}$ \\ ${ }^{1}$ School of Resources \& Civil Engineering, Northeastern University, Shenyang 110004, China \\ ${ }^{2}$ Highway and Bridge Engineering Institute, Dalian Maritime University, Dalian 116026, China \\ Correspondence should be addressed to Jiang Annan; jiangannan@163.com
}

Received 22 November 2012; Revised 10 February 2013; Accepted 23 February 2013

Academic Editor: Shengyong Chen

Copyright (C) 2013 Xing Jun et al. This is an open access article distributed under the Creative Commons Attribution License, which permits unrestricted use, distribution, and reproduction in any medium, provided the original work is properly cited.

Tunnel construction is a dynamic controlling system with observability and controllability; the feedback analysis requires identifying geophysics parameters and adjusting supporting parameters, and both of them are optimisation problems. The paper proposed a nonlinear optimization technique based on difference evolution arithmetic (DEA), least square support vector machine (LSSVM), and three dimensional numerical simulation. This method employs support vector machine with optimal architecture trained by the difference evolution arithmetic, instead of the time-consuming finite element analysis. Firstly, the three dimensional numerical simulation is used to create training and testing samples for LSSVM model construction. Then the nonlinear relationship between rock or anchoring parameters and displacement is constructed by support vector machine. Finally, the geophysics and supporting parameters are obtained by DE optimization arithmetic. The technique overcomes the conventional optimization method shortages of expending too much computing time and easily being limited in local optimal solution. This technique was verified by applying it to the feedback analysis of Dalian Metro in China, and the influence of the parameters of LSSVM and DE on the simulation ability of the algorithm was investigated.

\section{Introduction}

The geological body where the tunnel is constructed has uncertainty and complexity property, and there are two kinds of factors affecting the stability of surrounding rock. Some factors are inherent geophysical parameters of surrounding rock; the other factors are supporting parameters that human can adjust. The new Austrian tunnelling method points out the significance of surrounding rock displacements observation in construction process, and the posterior "information construction" and "observation construction" are also emphasizing the field monitoring information. Adjusting construction scheme based on identified rock mechanics parameters and obtaining the secure and economic scheme have important economic and scientific meaning.

The back analysis thinking is identifying rock parameters based on monitoring data, which had been firstly proposed by Sakurai and Takeuchi [1]. Mashimo summarized the update tunnel engineering techniques in Japan and proposed the concept of "redesign" [2]. Li proposed typical analogy analysis method and developed BMP program combining the techniques of rock classification, monitoring measurement, and rock mechanics analysis, which had gotten good application results [3]. Zhu and He had optimized construction subsequence of Xiao Langdi engineering taking broken zone as appraising index and fitness value; the calculation results stated that the method is feasible [4]. Construction schemes and parameters are not only affecting the tunnel safety but also deciding the economic cost. Arends proposed a method for the evaluation of tunnel safety using probabilistic risk assessment. The framework includes three criteria: personal, societal, and economic risk [5]. Pérez-Romero et al., and so forth, made joint use of geotechnical investigation campaigns, convergence measurements, and numerical simulations, thereby contributing towards the optimisation of the cross-section of the tunneled area support and lining [6].

Both rock parameters back analysis and supporting parameters adjusting are optimization problems in essence. Yuegeng Tang presented a nonlinear optimization technique (NOT) for conducting the back analyses of geotechnical 


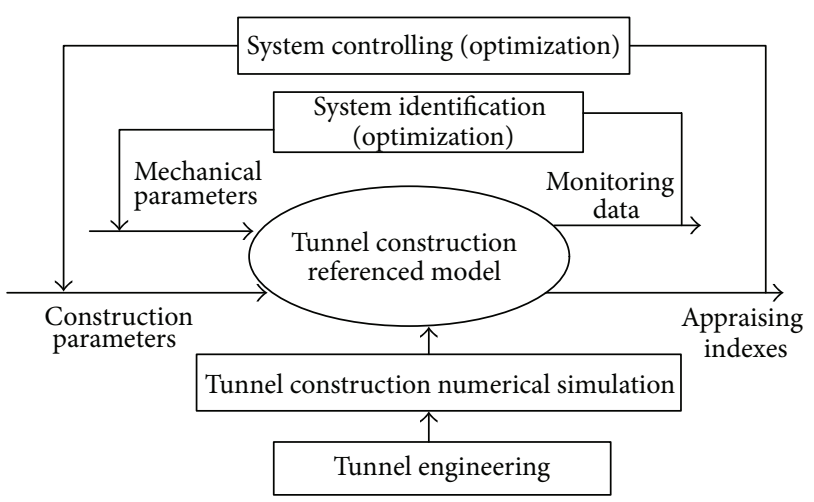

FIGURE 1: The adaptive control model of tunnel construction.

engineering problems based on the field observations. The developed NOT and additional auxiliary techniques are incorporated into a finite element code and then applied to the back analysis of excavation-induced wall deflection [7]. Hashash described and compared two inverse analysis approaches for an excavation project in downtown Chicago [8]. Along with the sensor techniques, tunnel monitoring developed from artificial, single information monitoring to automation, multiple information monitoring, genetic algorithm, and numerical method are also used in analysis of geotechnical problems [9-11]. The information science and intelligent methods are developed and applied in engineering [12-14] and overcome the shortcomings in conventional studies. Feng discussed how to establish the necessary quality and quantity of information required for rock engineering modelling and design [15]. Considering tunnel surrounding rock analysis being a complex nonlinear problem, the machine learning algorithms such as artificial neural network and support vector machine have been used in the rock displacement forecast or back analysis [16]. Jiang presented an integrated optimisation method for the feedback control of tunnel displacement; it combines the support vector machine (SVM), particle swarm optimisation (PSO), and 2D numerical analysis methods [17]. In that research, the method is applied only to control of the displacement objective and optimisation of the shotcrete parameters, ignoring the $3 \mathrm{D}$ space effect of tunnel heading face.

There are some problems in the tunnel construction optimization as follows. (1) Most methods are limited in surrounding rock parameters optimization and not including anchor parameters optimization. (2) Because the three dimensional numerical model expends too much time, the computing model is only limited in two dimensional space. (3) Because of the complex nonlinear characters of tunnel engineering, the conventional optimization method is easily limited in local optimum solution, and genetic algorithm has complex operation process and particle swarm optimization converges not steadily because of not having strictly convergence theory background. Aiming at the above problems, the paper constructed three dimensional feedback analysis method combining least square support vector machine (LSSVM) and difference evolution (DE) algorithm, and applied the method into a metro tunnel engineering of Dalian City in China.

\section{Tunnel Construction Nonlinear Optimization Method Based on DE-LSSVM}

2.1. Tunnel Construction Optimization Problem Statement. In fact, the tunnel dynamic construction process is an adaptive controlling problem. The process includes two optimization processes. First is system identification, that is, utilizing the measurement data to back analyze rock mechanics parameters; second is system controlling, that is, selecting the optimal construction schemes in order to ascertain the surrounding rock stability with certain economical cost. The adaptive control system model with multi-input and multioutput is proposed as Figure 1.

On one hand, construction process has high requirement for construction speed. On the other hand, however, numerical calculation is nonexplicit and calculation time is long, so the conventional optimization method is limited. Considering tunnel construction being a dynamic system which takes anchoring parameters and surrounding rock mechanics parameters as input variables and takes observing and stability indices as output indices, a nonlinear optimization technique combining LSSVM and DE algorithm is proposed as follows.

2.2. LSSVM Theory. SVM has been proposed for solving pattern recognition and function estimation problems in 1995 [18]. The SVM method is based on statistical learning theory and makes use of the principle of structural risk minimization, avoiding the extra learning problem of conventional learning method based on empirical risk minimization. Suykens proposed LSSVM which greatly simplifies the problem and has efficiency and accuracy in classifiers and regression $[19,20]$, LSSVM selects the different slack variable as the second norm of $\xi$, while the standard SVM adopts the $\xi$. Consider the problem of approximation in the set of $N$ samples and corresponding response is presented in

$$
\begin{array}{r}
D=\left\{\left(x^{1}, y^{1}\right),\left(x^{2}, y^{2}\right), \ldots,\left(x^{l}, y^{l}\right), \ldots,\left(x^{N}, y^{N}\right)\right\}, \\
x^{l} \in R^{n}, y^{l} \in R .
\end{array}
$$

The classifier model of primal space can be presented as follows:

$$
y(x)=\operatorname{sign}\left(w^{T} \varphi(x)+b\right) .
$$

The optimization problem in primal weigh space can be expressed as

$$
\underset{w, b, z}{\operatorname{MIN}} J(w, \varepsilon)=\frac{1}{2} W^{T} W+\frac{1}{2} \gamma \sum_{\mathrm{i}=1}^{N} \varepsilon_{1}^{2} .
$$

Such at

$$
y_{1}=w^{T} \varphi\left(x_{l}\right)+b+\varepsilon_{1}, \quad l=1,2, \ldots, N
$$


$\varphi(\cdot)=R^{n}-R^{n h}$ is a kernel that maps the input space into hyperspace (infinite space). $w \in R^{n}$ is weight vector in primal space, $\varepsilon_{1} \in R$ is error variable, and $b$ is bias term. The relative importance of ERM and SRM terms is determined by positive constant $\gamma$.

Then, according to (3), the Lagrangian description mapping to the SVM optimization problem is defined as follows:

$$
l(w, b, \varepsilon, a)=J(w, \varepsilon)-\gamma \sum_{i=0}^{n} a_{l}\left\{w^{T} \varphi\left(x_{\tau}\right)+b+\varepsilon_{l}-y_{l}\right\} .
$$

With Lagrangian multiplier $a$, the criterions for optimization problems can be written as

$$
\begin{gathered}
\frac{\partial(l(w, b, \varepsilon, a))}{\partial w}=0 \Longrightarrow w=\sum_{i=1}^{N} a_{l} \varphi\left(x_{l}\right) \\
\frac{\partial(l(w, b, \varepsilon, a)}{\partial b}=0 \Longrightarrow \sum_{i=1}^{N} a_{l}=0 \\
\frac{\partial(l(w, b, \varepsilon, a)}{\partial \varepsilon}=0 \Longrightarrow a_{l}=\gamma \varepsilon_{1} \\
\frac{\partial(l(w, b, \varepsilon, a)}{\partial a}=0 \Longrightarrow w^{T} \varphi\left(x_{l}\right)+b+\varepsilon_{l}-y_{l}=0 .
\end{gathered}
$$

After elimination of $w$ and $a$, the solution is obtained as

$$
\left[\begin{array}{cc}
0 & I^{T} \\
I & \Gamma+\frac{1}{\gamma} I
\end{array}\right]\left[\begin{array}{l}
b \\
a
\end{array}\right]=\left[\begin{array}{l}
0 \\
y
\end{array}\right]
$$

where $y=\left[y_{1}, y_{2}, \ldots, y_{N}\right], I=[1,1, \ldots, 1]$, and $a=$ $\left[a_{1}, a_{2}, \ldots, a_{N}\right]$.

Based on Mercer's condition, the kernel function can be expressed as

$$
K\left(x_{i}, x_{j}\right)=\varphi\left(x_{i}\right)^{T} \varphi\left(x_{j}\right), \quad \text { for } i, j=1,2, \ldots, N .
$$

The result LSSVM model for function estimation is obtained as follows:

$$
y(x)=\sum_{i=1}^{N} a_{i} K\left(x, x_{l}\right)+b .
$$

The optimization problem is translated to solving the linear equations by least square method; therefore, the LSSVM is named. Normally, the kernel function has three kinds: polynomial kernel function, radial basis kernel function, and sigmoid kernel function. The paper adopts radial basis kernel function as follows:

$$
k\left(x, x_{i}\right)=\exp \left(-\frac{\left|x-x_{i}\right|^{2}}{2 \sigma^{2}}\right) .
$$

2.3. The DE-LSSVM Nonlinear Model Describing Tunnel Construction. The anchoring parameters are controllable and surrounding rock parameters are inherent but not assured before excavation. Firstly, use monitoring data and anchoring parameters before heading face of tunnel to identify rock mechanics parameters. Then based on the identified rock mechanics parameters, optimize the anchoring parameters after heading face. The tunnel construction system can be described by numerical simulation; however, the three dimensional numerical simulation expends too much computing time; therefore, the LSSVM is introduced to replace the numerical simulation:

$$
\begin{gathered}
Y=\operatorname{LSSVM}(R), \quad Y=\operatorname{LSSVM}(M), \\
R=\left(r_{1}, r_{2}, r_{3}, \ldots, r_{a}\right), \\
M=\left(m_{1}, m_{2}, m_{3}, \ldots, m_{b}\right) \\
Y=\left(y_{1}, y_{2}, y_{3}, \ldots, y_{l}\right)
\end{gathered}
$$

where $Y$ is displacements of surrounding rock, $R$ and $M$ are rock mechanics parameters and anchoring parameters, respectively. $a$ is the number of rock mechanics variables, $b$ is the number of anchoring parameters, and $l$ is the number of outputting variables. Constructs orthogonal schemes of parameters combination and carries out three dimensional numerical simulation. Then obtains the data samples and trains support vector machine.

Some studies stated that different penal factor $c$ and kernel parameter $\sigma$ are corresponding to different LSSVM model and different forecast precision. LSSVM did not provide the theory of selecting the above parameters. DE optimization algorithm was proposed by Price and Storm of USA for solving Chebyshev Polynomial Polynominal Expression [21, 22]. It is simple, efficient and has good global optimization character [23]. The DE arithmetic is introduced and combined with LSSVM, and the DE-LSSVM model is constructed, which can solve the parameters selection problem of LSSVM. The DE-LSSVM algorithm computing process is as follows.

(1) Initial Population Generation. Get learning samples and test samples ready for LSSVM model, take penal factor and kernel parameter as two dimensional solution vector, and randomly generate $N_{P}$ two dimensional vectors according to the upper and lower boundaries, the formula is as follows:

$$
\begin{aligned}
X_{i j(0)}=\operatorname{rand} \times\left(x_{i j U}-x_{i j L}\right)+x_{i j L}, \\
\quad i=1,2, \ldots, N_{p} ; j=1,2,
\end{aligned}
$$

where $x_{i j U}, x_{i j L}$ are, respectively, upper and lower boundary of the $j$ component, and rand is the random between $[0,1] . N_{p}$ is population scale; the vector dimension number is corresponding to the number of parameters which to be optimized, in this state it is adopted as 2 .

(2) Mutation Operation. Reduce or magnify the error between arbitrary two vector individuals and add it to the third vector, in order to produce new variation 
variable. For the $G+1$ generation, the $j$ th component of the $i$ th variation vector is

$V_{i, j}(G+1)=x_{r 1 j}(G)+F \times\left(x_{r 2 j}(G)-x_{r 3 j}(G)\right)$,

where the suffixes $r 1, r 2, r 3$ are random integral numbers and different, and $F$ is scaling factor, which is used to adjust the step amplitude value of difference between vectors; the value is between 0 and 2 .

(3) Crossing Operation. Cross the objective vector $x_{i}(G)$ with variation vector $v_{i}(G+1)$, produce new sample vector $u_{i}(G+1)$, the $j$ th component is expressed as:

$$
u_{i j}(G+1)= \begin{cases}v_{i j}(G+1), & r_{j} \leq \mathrm{CR} \| j=\mathrm{rn}_{i} \\ x_{i j}(G), & r_{j}>\mathrm{CR}, \quad j \neq \mathrm{rn}_{i},\end{cases}
$$

where $r_{j} \in[0,1]$ is the random corresponding to the $j$ th component of vector, and CR $\in[0,1]$ is crossing probability constant. $\mathrm{rn}_{i}$ is random integral number selected from $1,2, \ldots, D$, which ensuring at least one component of $v_{i}(G+1)$ is used by $u_{i}(\mathrm{G}+1)$.

(4) Selection. Taking the sample vector as parameters of LSSVM, train LSSVM model by learning samples and forecast the test samples with the trained LSSVM model and take the maximal forecast error as the fitness value of difference evolution. Compare sample vector $u_{i}(G+1)$ with objective vector $x_{i}(G)$, if $u_{i}(G+$ $1)$ is responding to smaller objective function value, select $u_{i}(G+1)$, on the contrary, if $x_{i}(G)$ is responding to smaller objective function value, keep $x_{i}(G)$.

(5) Cyclic Iteration. Repeat the computation from (2) to (4), until $i$ has circulated from 1 to $\mathrm{Np}$, and $j$ has circulated from 1 to $n$; that is, one iteration of population has been finished. Circularly computing until the iteration number reaches the maximal step number or fitness value is less than the setting value, and the iteration is ended. Output the SVM parameters which is responding to the optimal LSSVM model expressing the data samples.

\subsection{Tunnel Construction Optimization Objective Functions} and Solving Procedure. The construction optimization procedure includes rock parameters identification and anchoring parameters optimization. For rock parameters identification: select a group of surrounding rock parameters and compute the surrounding rock displacements by the above trained LSSVM model, which compares with monitoring displacement data, until the error between computing and monitoring is minor enough, and the corresponding parameters are identified results. The LSSVM model is used to express the relation between surrounding rock parameters and monitoring displacements. For rock parameters identification, the constrained optimization problem is expressed by the following formula:

$$
\begin{aligned}
& \min \frac{1}{m} \sum_{i=1}^{m}\left(\left|\operatorname{LSSVM}_{i}(X)-Y_{i}^{0}\right|\right) \\
& \text { s.t } \quad X \in\left[X_{l}, X_{u}\right],
\end{aligned}
$$

where $Y_{i}^{0}$ is the observed value of surrounding rock, and $\operatorname{LSSVM}_{i}$ is the LSSVM forecast surrounding rock displacement corresponding to observation point $i . m$ is the number of observation points, $x_{i}$ is the $i$ th parameter, $n$ is the number of parameters, and $X_{l}$ and $X_{u}$ are, respectively, the lower and upper boundary of the solution vector.

For anchoring parameters optimization mainly based on the surrounding rock mechanics parameters identification, LSSVM model is also used to express the relation between anchoring parameters and surrounding rock displacements. The optimization function is as follows:

$$
\begin{aligned}
& \min \operatorname{Cost}(M) \\
& \text { s.t } \operatorname{LSSVM}_{j}(M)<Y_{j_{\max }}
\end{aligned}
$$

where $\operatorname{LSSVM}_{j}$ is the displacement forecast of surrounding rock control point $j$ corresponding to anchoring parameter M. $Y_{j \max }$ is the limit displacement corresponding to surrounding rock control point $j$. Cost $(M)$ is the supporting cost corresponding to the anchoring parameter $M$. The LSSVMS models in (15) and (16) were gotten by (9) and (10). And the objective functions are all solved by DE as follows.

(1) According to prior surveying and design information, respectively, assure the value scope of back analyzed rock mechanics parameter and anchoring parameter and construct calculation schemes by orthogonal test and uniformity test theory.

(2) Construct three dimensional numerical model and calculate. Carry out numerical simulation for each parameters combination and obtain monitoring information of key points corresponding to each parameters combination, which becomes a learning sample.

(3) Search the optimal support vector machine parameters by difference evolution.

(4) Adopting the optimized parameters and training the support vector machine with the above learning samples, obtain the DE-LSSVM nonlinear model mapping the relation between mechanics parameters (or anchoring parameters) and rock displacements.

(5) Substitute the above nonlinear mapping model and monitoring displacement in the objective function of (15). Search the identification parameters by difference evolution algorithm.

(6) Based on the identified parameters, take anchoring parameters as variables, repeat (2)-(4), and take formula (16) as objective function, optimized anchoring parameters by DE again. The feedback analysis process of tunnel construction based on DE-LSSVM is shown in Figure 2.

\section{Engineering Application}

3.1. Engineering Introduction. Applied the method to Dalian Metro 1\# line tunnel engineering, and studied the running tunnel from Xueyuan Square to Dalian Maritime University. 


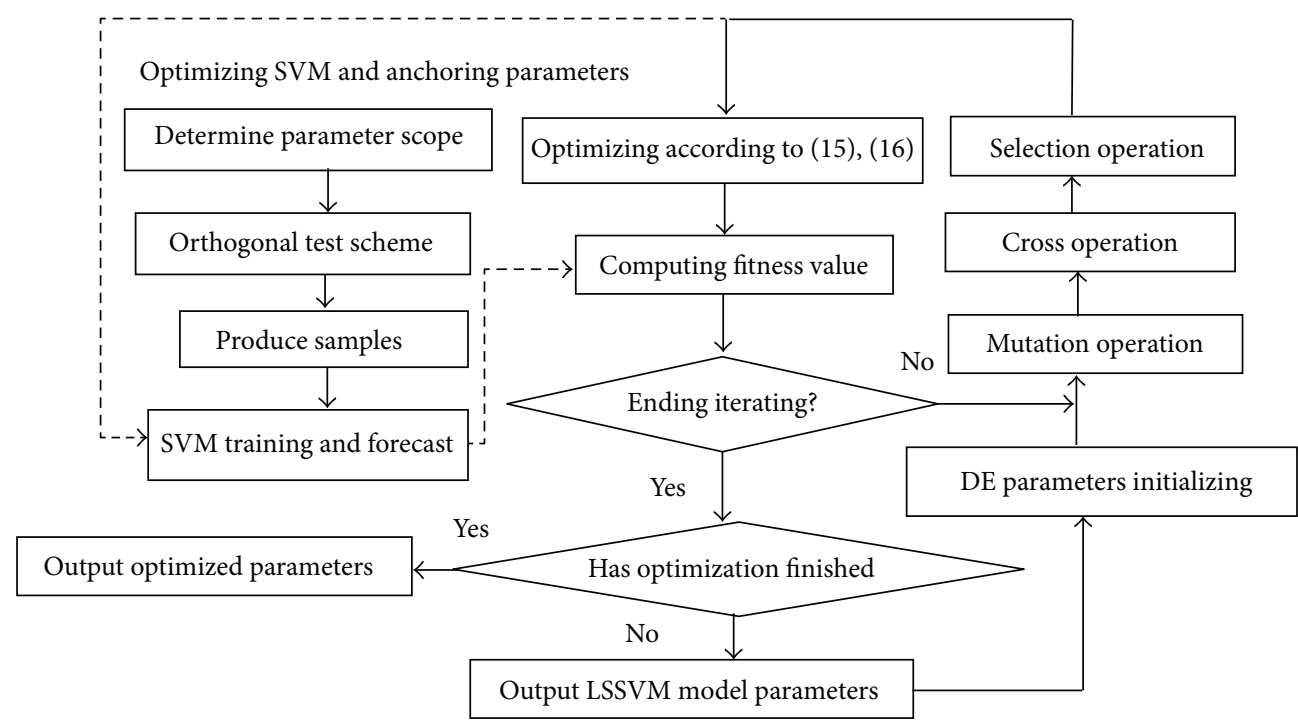

FIGURE 2: Tunnel construction optimization solving procedure based on DE-LSSVM.

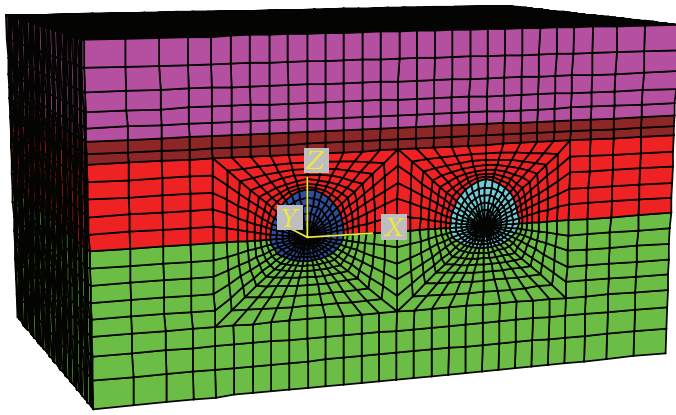

(a) Tunnel three dimensional numerical model

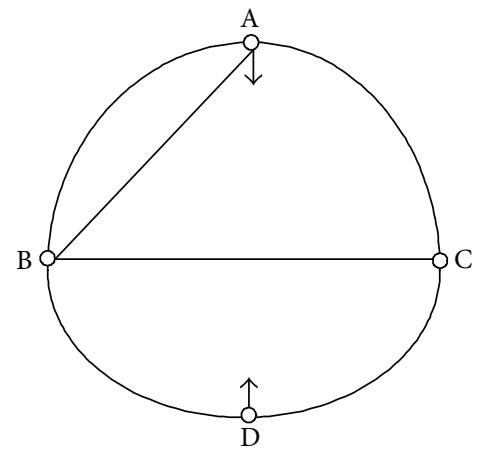

(b) Measuring points arrangement of section

FIGURE 3: The three dimensional numerical model and monitoring section.

The geological strata are, respectively, artificial accumulation horizon of quaternary Holocene Series $\left(Q_{4}{ }^{\mathrm{mL}}\right)$, quaternary Alluvium and Diluvium $\left(Q_{4}{ }^{\mathrm{al}+\mathrm{pl}}\right)$, and Changlinzi group slate of Sinian system (Zwhc). The tunnel belongs to super shallow buried tunnel and the the distance from arc top to surface is 12 meters and the surrounding rock is IV class. According to the original design scheme, the tunnel has the width of $5.6 \mathrm{~m}$, height of $6 \mathrm{~m}$ and adopts system grouted bolts. The bolt has length of $3.0 \mathrm{~m}$, diameter of $22 \mathrm{~mm}$, and space of $1 \mathrm{~m}$; the thickness of shotcrete layer is $30 \mathrm{~cm}$. It adopts benching tunneling construction method. The study region is from $\mathrm{AK} 19+288$ to $\mathrm{AK} 19+318$ of the mileage and there are two different strata slates with different weathering degree surrounding the tunnel.

The three dimensional numerical model is constructed and the model adopts artesian coordinate system and has 71859 nodes and 70000 elements, and the calculation scope is $45.8 \mathrm{~m} \times 30 \mathrm{~m} \times 29.8 \mathrm{~m}$. The $x$ direction is vertical to the main tunnel axis, $z$ direction is vertical, and $y$ direction is along the main tunnel axis. The bolts are simulated by cable elements and shotcrete layer is simulated by shell elements. The null model is adopted for excavation and Mohr-Coulomb yield criterion is used for plastic damage. The model is shown in Figure 3(a). In the construction process, the typical monitoring section is shown in Figure 3(b). The convergence lines of $\mathrm{AB}$ and $\mathrm{BC}$, and the sedimentation of $\mathrm{A}$ and rising of $\mathrm{D}$ should be monitored.

3.2. Rock Mechanics Parameters Identification. According to prior explore and laboratory tests, the self-weight stress is adopted, $\sigma_{x}=k * \sigma_{z}, \sigma_{x}$ is horizontal ground stress, and $\sigma_{z}$ is vertical groundstress. $k$ is side pressure coefficient; the surrounding rock parameters are Young's modulus and Poisson ratio. The scope of rock mechanics parameters are Young's modulus of serious weathered slate $E_{1}$ that adopts 20 100 MPa, Poisson ratio of serious weathered slate $u_{1}$ that adopts $0.27 \sim 0.35$, and Young's modulus of middle weathered 

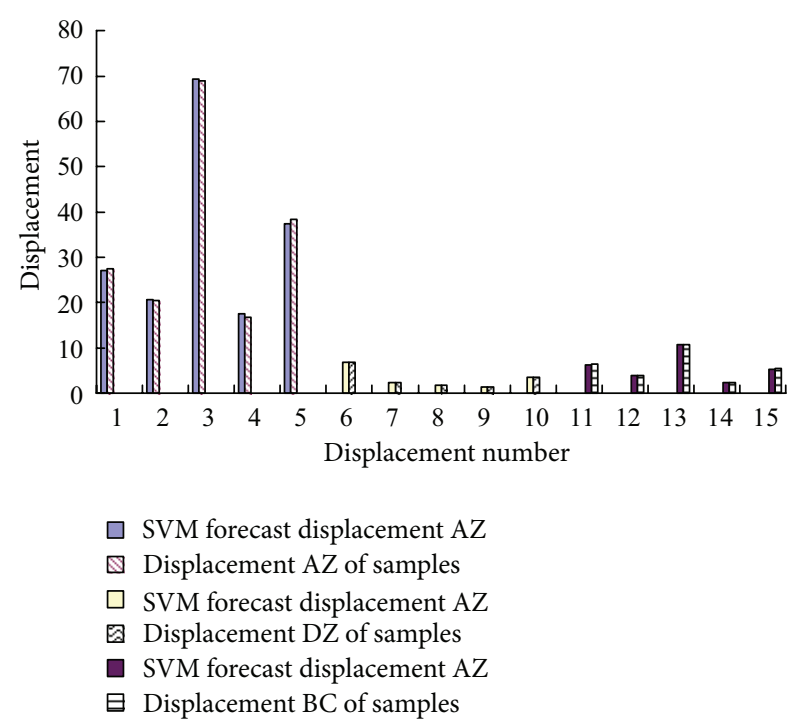

FIgURE 4: The forecast effect test of $\operatorname{LSSVM}(C=23.5, \delta=56)$.

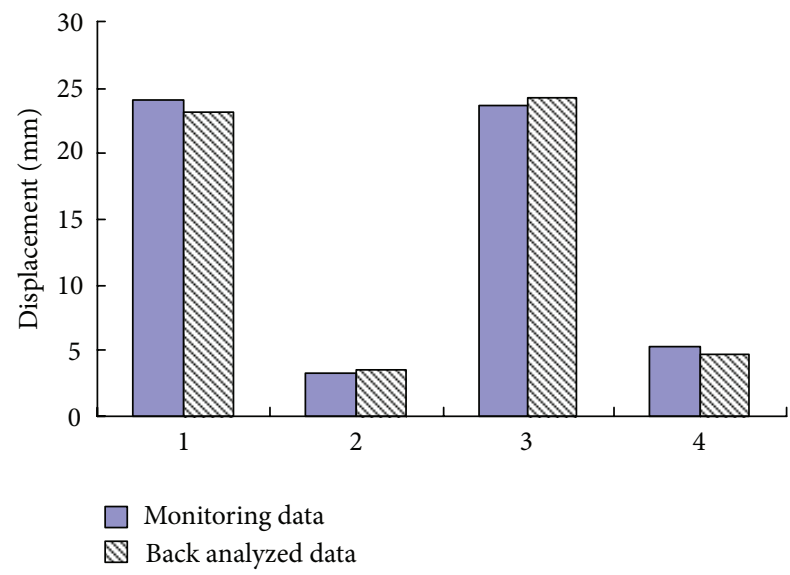

FIGURE 5: Optimized calculation and monitoring displacement.

slate $E_{2}$ that adopts $0.2 \sim 1 \mathrm{GPa}$, Poisson ratio ofmiddle weathered slate $u_{2}$ that adopts $0.23 \sim 0.31$. The monitoring indices are arc top sedimentation $\mathrm{AZ}$, the rising of bottom DZ, and the convergence displacements of $\mathrm{AB}$ and $\mathrm{BC}$. Selected orthogonal design table of $L_{25}\left(5^{6}\right)$ and uniform design table of $U 5 *\left(5^{4}\right)$ for $E_{1}, u_{1}, E_{2}$, and $u_{2}$ between parameters scope, which construct 25 orthogonal schemes and 5 uniform schemes, carried out numerical simulation and obtained 25 learning samples and 5 test samples. The learning samples are shown in Table 1. Through the DE-LSSVM calculation as 2.3 , the LSSVM has been trained by inputting samples, and the nonlinear mapping models between surrounding rock parameters and monitoring displacements are obtained. Figure 4 shows the forecast error of LSSVM model for the test samples, and it can be found that the error is minor and

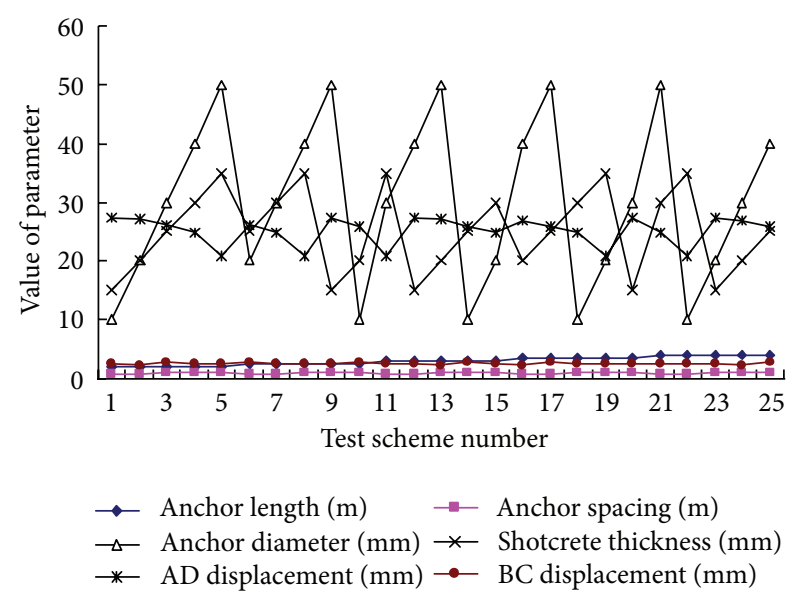

Figure 6: The parameters and displacements of orthogonal schemes.

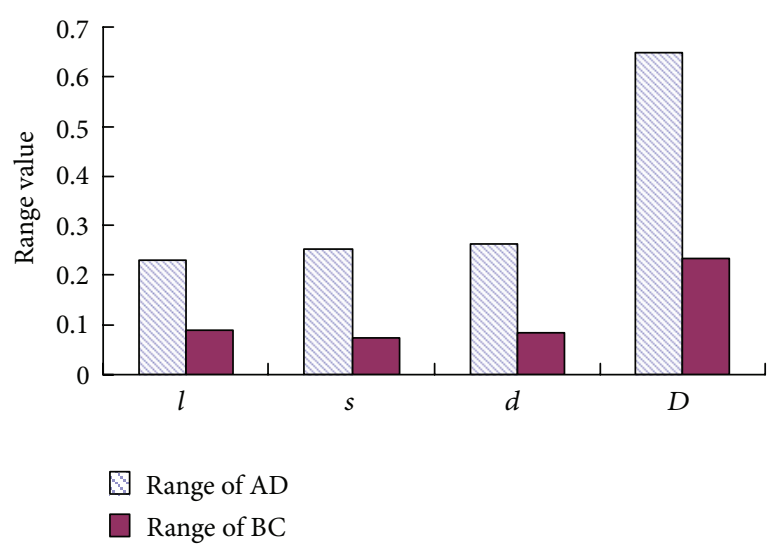

FIGURE 7: The sensibility analysis of anchoring parameters.

the trained LSSVM model can reflect the relation between mechanics parameters and displacements well.

The monitored displacements are as follows: $\mathrm{AZ}$ is $24.0 \mathrm{~mm}, \mathrm{DZ}$ is $3.2 \mathrm{~mm}, \mathrm{AB}$ is $23.6 \mathrm{~mm}$, and $\mathrm{BC}$ is $5.4 \mathrm{~mm}$. Substitute the monitored data to formula (15), using above trained LSSVM model, which identified the surrounding rock mechanics parameters by $\mathrm{DE}$ arithmetic. It is shown form Figure 5 that the trained LSSVM model has well forecast ability. The DE original parameters are set as follows. The number of optimal variables is 4 , the population scale is 20 , and the iteration number is 500 . While the DE curve converges, the corresponding optimal mechanics parameters $E_{1}, E_{2}, u_{1}$, and $u_{2}$ are $58.6 \mathrm{Mpa}, 347 \mathrm{MPa}, 0.31$, and 0.27 , respectively.

In order to verify the rationality of the identified parameters, which inputted the identified parameters to the three dimensional numerical model and carried out calculation with finite difference method, the comparison between calculated results and monitoring data is shown in Figure 5. The maximal relative error between feedback calculated value and monitoring data is only $9.375 \%$, and the maximal absolute error is $0.90 \mathrm{~mm}$; therefore, the accuracy is satisfied. 


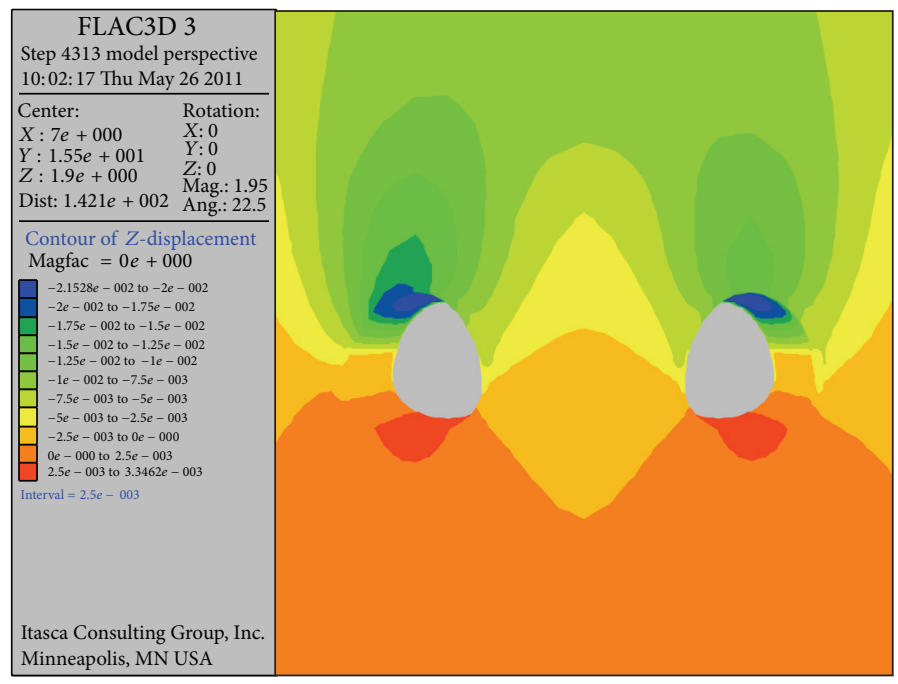

(a) The original scheme

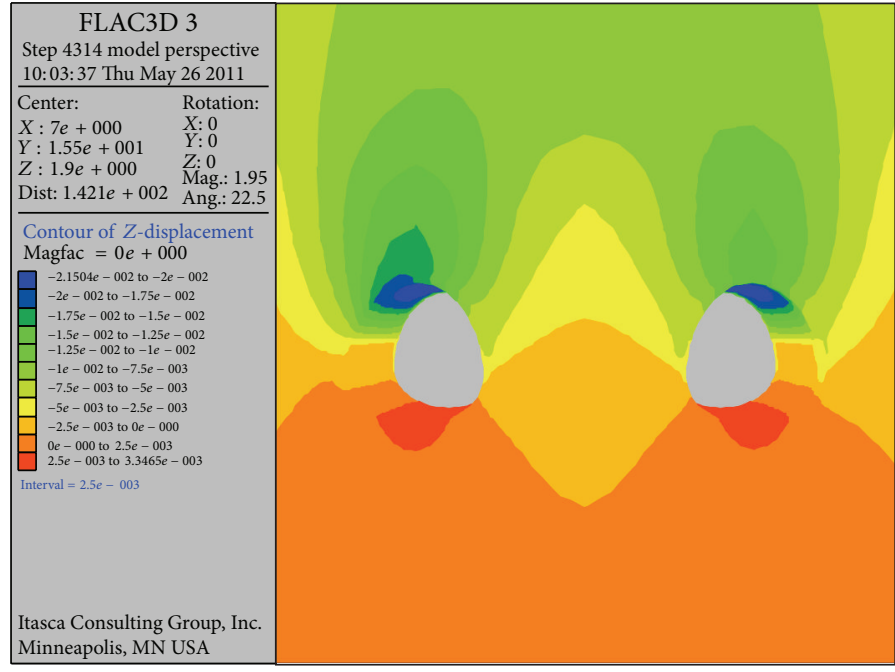

(b) The optimized scheme

FIGURE 8: The Z-displacement contour comparison between original and optimized schemes.

3.3. Anchoring Parameters Optimization. Cable and shotcrete layer are main reinforcement measures for controlling the surrounding rock stability of the tunnel. Based on the identified rock mechanics parameters of surrounding rock, the anchoring parameters should be optimized according to formula (16). The parameters to be optimized in this study are cable length $l$ with the range of $2.0 \sim 4.0 \mathrm{~m}$, cable diameter $d$ with the range of $10 \sim 50 \mathrm{~mm}$, cable space $s$ with the range of $0.7 \sim 1.1 \mathrm{~m}$, and shotcrete thickness $D$ with the range of $15 \sim 35 \mathrm{~cm}$. According to the orthogonal design table of $L_{25}\left(5^{6}\right)$ and the uniform design table of $U 5 *\left(5^{4}\right)$, which arrange numerical test, take calculation results of 25 orthogonal schemes as learning samples and take calculation results of 5 uniform schemes as test samples.

Calculated above supporting schemes using the constructed three dimensional numerical model, and used the displacements of $\mathrm{AD}$ and $\mathrm{BC}$ as surrounding rock stability indices. The anchoring parameters combination and the corresponding displacements of $\mathrm{AD}$ and $\mathrm{BC}$ are shown in Figure 6. Based on the above calculation results, the parameters were studied by the range analysis, and the sensibility of the anchoring parameters is shown by Figure 7. It is shown from Figure 7 that the sequence of the parameters sensibility affecting surrounding rock displacements $\mathrm{AD}$ and $\mathrm{BC}$ are $D, l, d$, and $s$ in turn.

Set the optimized variables as $M=\left\{m_{1}, m_{2}, m_{3}, m_{4}\right\}$, the length of cable is $m_{1}$, the space of cables is $m_{2}$, the diameter of cable is $m_{3}$, and the thickness of shotcrete is $m_{4}$. The optimization objective is the minimal supporting cost according to the stability condition that is, displacement condition, just expressed as formula (16). The nonlinear relation of LSSVM $\mathrm{M}_{j}$ between anchoring parameters and surrounding rock displacements has been trained by orthogonal schemes dataset. The relation between anchoring parameters and cost 
can be pressed by experience calculation formula. Considering the safety demand set the limit displacement of $\mathrm{AD}$ is $25 \mathrm{~mm}$, and the limit displacement of BC is $2.6 \mathrm{~mm}$. Using DE arithmetic to optimize the anchoring parameters, set the original parameters as follows: the number of optimization variables is 4 , the population scale is 20 , and the iteration number is 500 .

Run the DE-LSSVM optimization program, while the curve has converged; the optimal anchoring parameters are obtained. The optimized scheme, original design scheme, and part of the orthogonal schemes are all listed in Table 2.

The optimized supporting parameters are anchor length of $2.3 \mathrm{~m}$, anchors space of $0.97 \mathrm{~m}$, anchor diameter of $19 \mathrm{~mm}$, and shotcrete thickness of $30 \mathrm{~cm}$. The corresponding supporting cost is 10482.61 Yuan each running meter and economized 1382.76 Yuan each running meter. For the interval tunnel is about 1000 meters, the saved cost is about 1382760 Yuan. Input the optimized supporting parameters into the numerical model and carried out the simulation. Figure 8 is The Z-displacement contour comparison between original and optimized schemes. There are minor difference between the $\mathrm{Z}$ displacements of the two schemes, that means that the optimized scheme also ensures the tunnel stability well.

\section{Discussion}

4.1. Kernel Parameters Affecting LSSVM Model. Reflecting relation between rock mechanics parameters (or anchoring parameters) and surrounding rock displacements is a key problem affecting the optimization result. In the above optimisation process, the kernel parameter $\sigma$ and the penal coefficient $c$ are important factors that affect the generational performance of LSSVM. The effects of LSSVM parameters on the forecast result are shown in Figure 9.

The $x y$ coordinates in Figure 9 are, respectively, the kernel parameter, $\sigma$ and the penal factor, $c$, and the $z$ coordinate is relative forecast error. For values of $c$ below 20, the magnitude of the forecast error approaches 0.2 ; when $c$ increases to more than 20, the corresponding forecast error is reduced to 0.05 , while $c$ increases to more than 30 , the corresponding forecast error has increased more than 0.2 . When $\sigma$ is less than 40 and more than 60 , the magnitude of the corresponding forecast errors are 0.2 and 0.1 , respectively. And when $\sigma$ is about 56, the corresponding forecast error is reduced to 0.047 . Variations in the penal factor, $c$, and the kernel parameter, $\sigma$, will change the LSSVM forecast error, which shows that it is imperative to select proper parameters to guarantee the forecast performance. Because LSSVM does not have a method for selecting these parameters, optimizing parameters through DE arithmetic can avoid blind parameter selection. Therefore, the proposed DE-LSSVM method has high computing efficiency for LSSVM parameter selection.

4.2. DE Optimization Convergence Property. Compare the proposed optimization method with that of literature 17, firstly, three dimensional numerical model has been used to simulate tunnel, which can fully reflect the space effect of tunnel heading face, overcoming the shortage of two dimensional

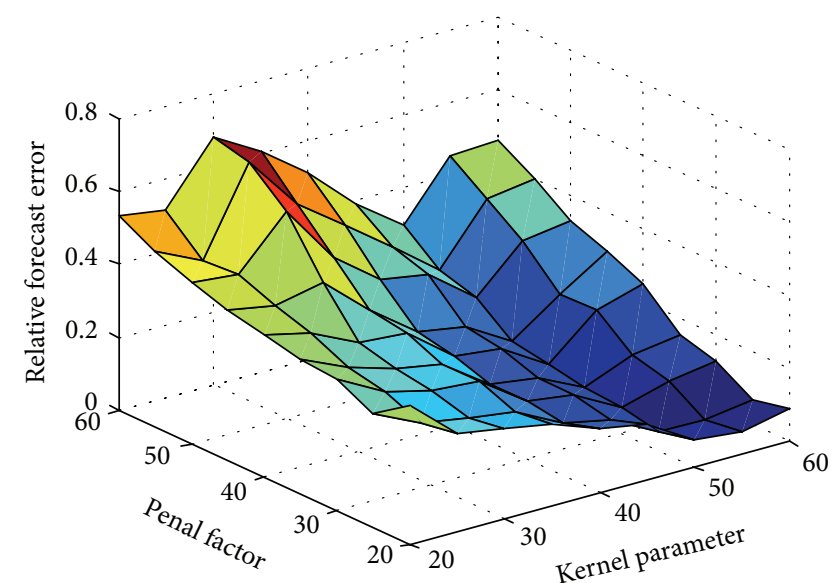

FIGURE 9: The forecast relative error changing according to $C$ and $\sigma$.

model. Secondly, in this study the parameters of shotcrete thickness and cables have been synthetically optimized using surrounding rock displacements as constraint conditions and economic indices as objective function, which is more reasonable than only considering the shortcrete parameter and displacement controlling. Finally, the DE algorithm is selected replacing the PSO algorithm in this study.

In the optimization process of $\mathrm{DE}$, different scaling factor $F$ and crossing probability constant CR will affect the arithmetic converging property. For the examples of identifying surrounding rock mechanics parameters above, while $F$ equates $0.8, \mathrm{CR}$ changes from 0.5 to 0.9 , and the corresponding converging curves are shown in Figure 10.

It is shown that while CR equates 0.5 , the converging property is optimal; it has converged at about 100 step. Next CR adopted $0.7,0.9$, and 0.6 in order. It converges slowly while CR equates 0.8 , and the corresponding converging step is 120 . As a whole, the curves of different parameters can converge quickly and the searching property of DE arithmetic is good. While DE curve converges, the corresponding solution is the optimized parameters.

Figure 11 shows the converging curves of DE, PSO, and improved PSO (IPSO) in the optimizing process of identifying surrounding rock mechanics parameters. It is shown that for the optimzing case the converging property of the DE is better than normal PSO and IPSO. Because the DE arithmetic has more strict math theory background than PSO, it has better converging speed and optimizing property. Totally, it is observed that the proposed optimizing technique has more advantages than the former techniques.

\section{Conclusion}

The study presented a nonlinear optimization technique of tunnel construction based on DE, LSSVM, and three dimensional numerical simulation. This method can be divided into two main sections: feedback analysis of the surrounding rock mechanical parameters and optimisation of support parameters. The method only took use of scheme 
TABLE 1: Orthogonal scheme and calculated displacements.

\begin{tabular}{|c|c|c|c|c|c|c|c|c|}
\hline Factor & $E_{1}(\mathrm{MPa})$ & $u_{1}$ & $E_{2}(\mathrm{GPa})$ & $u_{2}$ & $\mathrm{AZ}(\mathrm{mm})$ & $\mathrm{DZ}(\mathrm{mm})$ & $\mathrm{AB}(\mathrm{mm})$ & $\mathrm{BC}(\mathrm{mm})$ \\
\hline 1 & 20 & 0.27 & 0.2 & 0.23 & 70.05 & 7.137 & 67.78 & 10.795 \\
\hline 2 & 20 & 0.29 & 0.4 & 0.25 & 69.50 & 3.541 & 66.74 & 10.050 \\
\hline 3 & 20 & 0.31 & 0.6 & 0.27 & 69.18 & 2.341 & 66.13 & 10.230 \\
\hline 4 & 20 & 0.33 & 0.8 & 0.29 & 68.91 & 1.740 & 65.54 & 10.806 \\
\hline 5 & 20 & 0.35 & 1.0 & 0.31 & 68.65 & 1.377 & 64.92 & 11.537 \\
\hline 6 & 40 & 0.27 & 0.4 & 0.27 & 38.30 & 3.498 & 36.94 & 5.596 \\
\hline 7 & 40 & 0.29 & 0.6 & 0.29 & 38.07 & 2.312 & 36.52 & 5.503 \\
\hline 8 & 40 & 0.31 & 0.8 & 0.31 & 37.91 & 1.715 & 36.22 & 5.614 \\
\hline 9 & 40 & 0.33 & 1.0 & 0.23 & 37.72 & 1.408 & 36.00 & 5.675 \\
\hline 10 & 40 & 0.35 & 0.2 & 0.25 & 38.48 & 6.985 & 36.73 & 8.816 \\
\hline 11 & 60 & 0.27 & 0.6 & 0.31 & 26.73 & 2.288 & 25.75 & 3.839 \\
\hline 12 & 60 & 0.29 & 0.8 & 0.23 & 26.55 & 1.763 & 25.48 & 3.641 \\
\hline 13 & 60 & 0.31 & 1.0 & 0.25 & 26.45 & 1.398 & 25.27 & 3.785 \\
\hline 14 & 60 & 0.33 & 0.2 & 0.27 & 27.30 & 6.941 & 26.23 & 6.554 \\
\hline 15 & 60 & 0.35 & 0.4 & 0.29 & 26.68 & 3.438 & 25.36 & 5.531 \\
\hline 16 & 80 & 0.27 & 0.8 & 0.25 & 20.58 & 1.752 & 19.83 & 2.745 \\
\hline 17 & 80 & 0.29 & 1.0 & 0.27 & 20.49 & 1.390 & 19.66 & 2.836 \\
\hline 18 & 80 & 0.31 & 0.2 & 0.29 & 21.43 & 6.898 & 20.71 & 5.392 \\
\hline 19 & 80 & 0.33 & 0.4 & 0.31 & 20.79 & 3.412 & 19.85 & 4.382 \\
\hline 20 & 80 & 0.35 & 0.6 & 0.23 & 20.47 & 2.333 & 19.44 & 3.832 \\
\hline 21 & 100 & 0.27 & 1.0 & 0.29 & 16.79 & 1.381 & 16.17 & 2.261 \\
\hline 22 & 100 & 0.29 & 0.2 & 0.31 & 17.79 & 6.846 & 17.28 & 4.763 \\
\hline 23 & 100 & 0.31 & 0.4 & 0.23 & 17.05 & 3.508 & 16.36 & 3.295 \\
\hline 24 & 100 & 0.33 & 0.6 & 0.25 & 16.82 & 2.321 & 16.04 & 3.122 \\
\hline 25 & 100 & 0.35 & 0.8 & 0.27 & 16.67 & 1.726 & 15.83 & 3.095 \\
\hline
\end{tabular}

TABLE 2: The results of optimization compared with original and orthogonal schemes.

\begin{tabular}{lccccccc}
\hline & $\begin{array}{c}\text { Anchor } \\
\text { length } \\
(\mathrm{m})\end{array}$ & $\begin{array}{c}\text { Anchors } \\
\text { space } \\
(\mathrm{m})\end{array}$ & $\begin{array}{c}\text { Anchor } \\
\text { diameter } \\
(\mathrm{mm})\end{array}$ & $\begin{array}{c}\text { Shotcrete } \\
\text { thickness } \\
(\mathrm{cm})\end{array}$ & $\begin{array}{c}\text { AD } \\
(\mathrm{mm})\end{array}$ & $\begin{array}{c}\text { BC } \\
(\mathrm{mm})\end{array}$ & $\begin{array}{c}\text { Supporting cost } \\
(\text { Yuan })\end{array}$ \\
\hline Optimized value & 2.3 & 0.97 & 19 & 30 & 24.83 & 2.53 & 10482.61 \\
Original scheme & 3.0 & 1.0 & 22 & 30 & 24.80 & 2.52 & 11865.37 \\
Scheme 4 & 2.0 & 1.0 & 40 & 30 & 24.83 & 2.53 & 26063.87 \\
Scheme 8 & 2.5 & 0.9 & 40 & 35 & 20.89 & 2.59 & 35783.16 \\
Scheme 19 & 3.5 & 1.0 & 20 & 35 & 20.86 & 2.58 & 14684.19 \\
\hline
\end{tabular}

samples calculated by three dimensional numerical simulation; it employs LSSVM with optimal architecture trained by the difference evolution arithmetic, instead of the timeconsuming finite element analysis, overcoming the shortages of expending too much time and easily being limited in local optimization solution of conventional back analysis method.

The three dimensional numerical model was used to simulate tunnel, which can fully reflect the space effect of tunnel heading face, overcoming the shortage of two dimensional model. Representative samples for LSSVM training and factor sensitivity analysis were given by the orthogonal experimental design method, which efficiently reduced the number of numerical simulations. The LSSVM method was based on statistical learning theory and made use of the principle of structural risk minimization, overcoming empirical risk minimization of conventional statistic learning method (such as artificial neural network) and improving the generalizing ability for limited learning samples.

This study provided a real time, quantitative and powerful means to inform construction activities and can adjust construction schemes dynamically. Because LSSVM does not have a method for selecting these parameters, optimizing LSSVM parameters through DE arithmetic can avoid blind parameter selection. The parameters of lining and cables were synthetically optimized using surrounding rock displacements as constraint conditions and economic indices as objective function, which was more reasonable than former methods. DE converged quickly and had good 


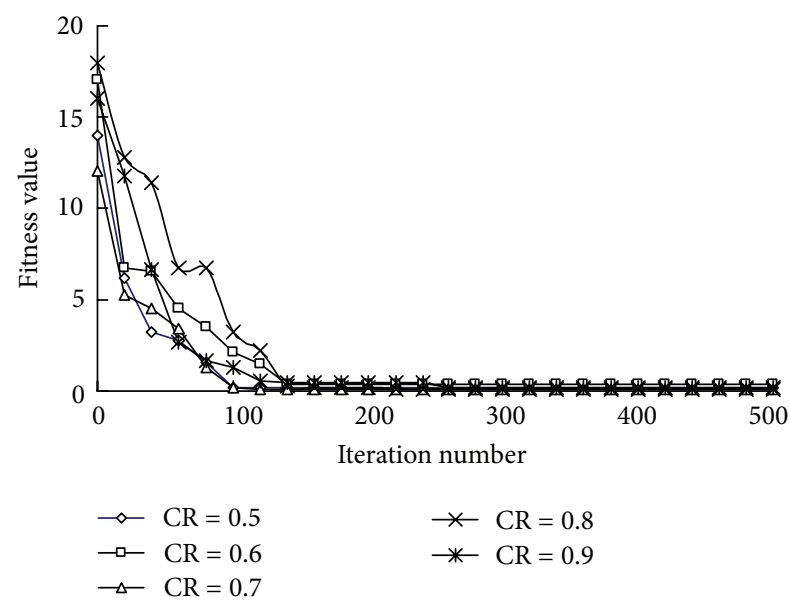

Figure 10: The DE converging curve $(F=0.8)$.

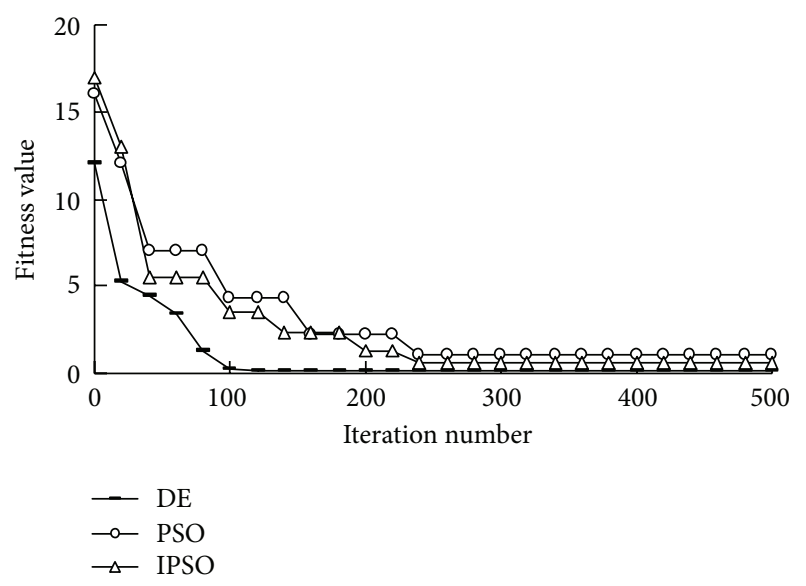

FIGURE 11: The converging curves of different algorithms.

global optimization characters, guaranteeing the rationality of the parameters identification and anchoring parameters optimization. The case study demonstrated that the displacements predicted by the identified parameters were in good agreement with field measurements and that the obtained supporting parameters were an acceptable control for achieving the stability and economic objective.

\section{Acknowledgments}

The authors deeply appreciate support from Fundamental Research Funds for the Central Universities (2011JC012, 2012TD015), National Natural Science Foundation (5107 9010), and National "Twelfth Five-Year" Plan for Science and Technology Support (2012BAJ17B01, 2012BAJ17B02).

\section{References}

[1] S. Sakurai and K. Takeuchi, "Back analysis of measured displacements of tunnels," Rock Mechanics and Rock Engineering, vol. 16, no. 3, pp. 173-180, 1983.
[2] H. Mashimo, "State of the road tunnel safety technology in Japan," Tunnelling and Underground Space Technology, vol. 17, no. 2, pp. 145-152, 2002.

[3] S. H. Li, New Theory of Tunnel Supporting: The Application and Theory of Typical Similar Supporting Design, China Science Press, Beijing, China, 1999.

[4] W. S. Zhu and M. C. He, Surrounding Rock Stability of Complex Condition and Rock Mass Dynamic Construction Mechanics, China Science Press, Beijing, China, 1995.

[5] B. J. Arends, S. N. Jonkman, J. K. Vrijling, and P. H. A. J. M. van Gelder, "Evaluation of tunnel safety: towards an economic safety optimum," Reliability Engineering and System Safety, vol. 90, no. 2-3, pp. 217-228, 2005.

[6] J. Pérez-Romero, C. S. Oteo, and P. de la Fuente, "Design and optimisation of the lining of a tunnel in the presence of expansive clay levels," Tunnelling and Underground Space Technology, vol. 22, no. 1, pp. 10-22, 2007.

[7] Y. G. Tang and G. T. C. Kung, "Application of nonlinear optimization technique to back analyses of deep excavation," Computers and Geotechnics, vol. 36, no. 1-2, pp. 276-290, 2009.

[8] Y. M. A. Hashash, S. Levasseur, A. Osouli, R. Finno, and Y. Malecot, "Comparison of two inverse analysis techniques for learning deep excavation response," Computers and Geotechnics, vol. 37, no. 3, pp. 323-333, 2010.

[9] S. C. Lim, C. H. Eab, K. H. Mak, M. Li, and S. Y. Chen, "Solving linear coupled fractional differential equations by direct operational method and some applications," Mathematical Problems in Engineering, vol. 2012, Article ID 653939, 28 pages, 2012.

[10] H. L. Liu, P. Li, and J. Y. Liu, "Numerical investigation of underlying tunnel heave during a new tunnel construction," Tunnelling and Underground Space Technology, vol. 26, no. 2, pp. 276-283, 2011.

[11] F. S. Zhang, X. X. Xie, and H. W. Huang, "Application of ground penetrating radar in grouting evaluation for shield tunnel construction," Tunnelling and Underground Space Technology, vol. 25, no. 2, pp. 99-107, 2010.

[12] Y. Xu, X. Y. Chen, and Q. H. Li, "INS/WSN-integrated navigation utilizing LSSVM and $H_{\infty}$ filtering," Mathematical Problems in Engineering, vol. 2012, Article ID 707326, 19 pages, 2012.

[13] P. Z. Lu, S. C. Chen, and Y. J. Zheng, "Artificial intelligence in civil engineering," Mathematical Problems in Engineering, vol. 2012, Article ID 145974, 22 pages, 2012.

[14] C. Carlo, S. Y. Chen, and A. Gani, "Information and modeling in complexity," Mathematical Problems in Engineering, vol. 2012, Article ID 868413, 4 pages, 2012.

[15] X. T. Feng, Z. Zhang, and Q. Sheng, "Estimating mechanical rock mass parameters relating to the Three Gorges Project permanent shiplock using an intelligent displacement back analysis method," International Journal of Rock Mechanics and Mining Sciences, vol. 37, no. 7, pp. 1039-1054, 2000.

[16] A. N. Jiang, "Forecasting nonlinear time series of surrounding rock deformations of underground cavern based on PSO-SVM," Rock and Soil Mechanics, vol. 28, no. 6, pp. 1176-1180, 2007 (Chinese).

[17] A. N. Jiang, S. Y. Wang, and S. L. Tang, "Feedback analysis of tunnel construction using a hybrid arithmetic based on Support Vector Machine and Particle Swarm Optimisation," Automation in Construction, vol. 20, no. 4, pp. 482-489, 2011.

[18] V. N. Vapnik, The Nature of Statistical Learning Theory, Springer, New York, NY, USA, 1995. 
[19] J. A. K. Suykens, T. van Gestel, and J. de Brabanter, Least Squares Support Vector Machines, World Scientific, Singapore, 2002.

[20] J. A. K. Suykens and J. Vandewalle, "Least squares support vector machine classifiers," Neural Processing Letters, vol. 9, no. 3, pp. 293-300, 1999.

[21] R. Storn and K. Price, "Differential evolution," 2008, http:// wwwl.icsi.berkeley.edu/ storn/code.html.

[22] A. W. Mohamed, H. Z. Sabry, and M. Khorshid, "An alternative differential evolution algorithm for global optimization," Journal of Advanced Research, vol. 3, no. 2, pp. 149-165, 2012.

[23] J. Ilonen, J. K. Kamarainen, and J. Lampinen, "Differential evolution training algorithm for feed-forward neural networks," Neural Processing Letters, vol. 17, no. 1, pp. 93-105, 2003. 


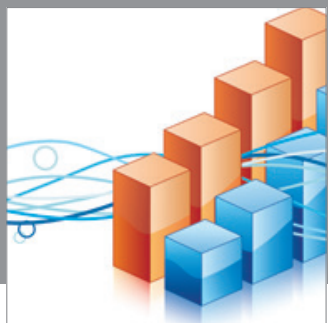

Advances in

Operations Research

mansans

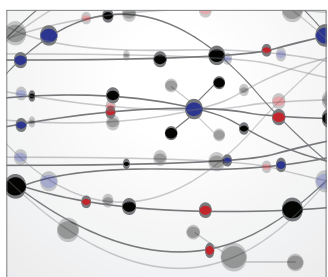

The Scientific World Journal
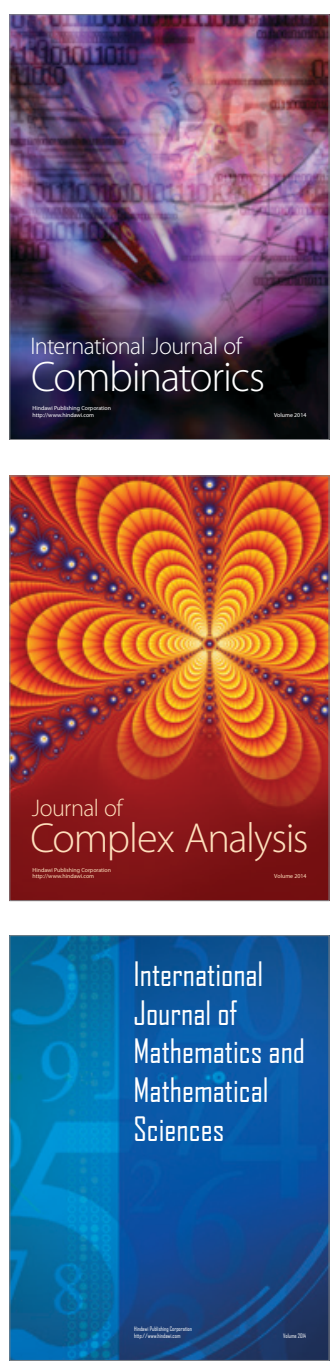
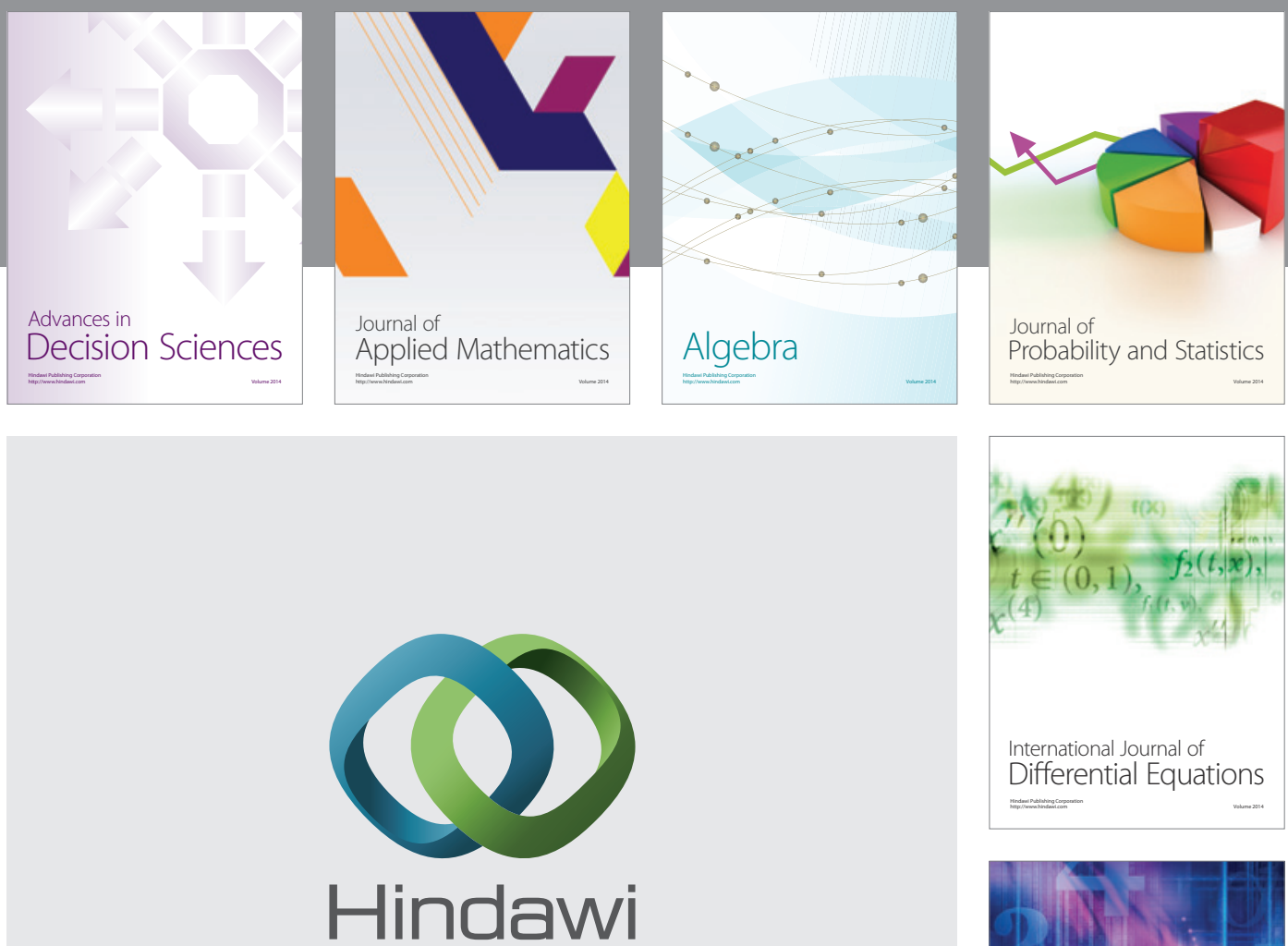

Submit your manuscripts at http://www.hindawi.com
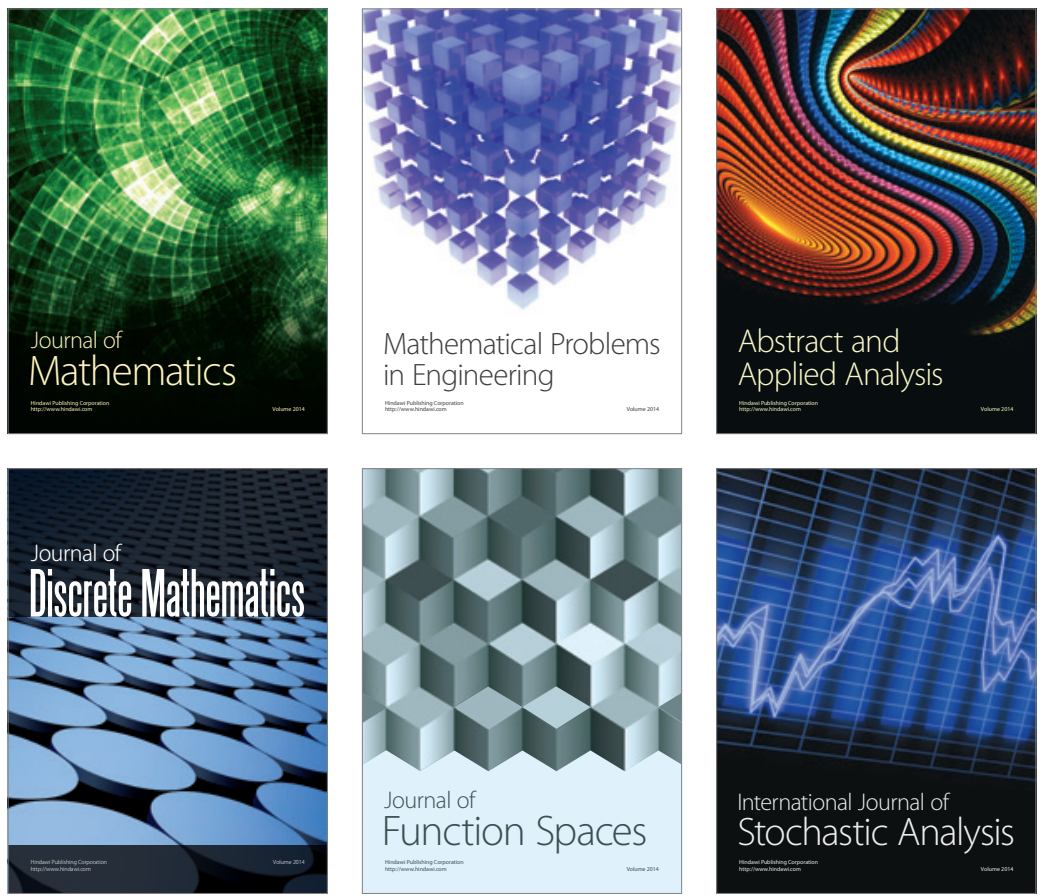

Journal of

Function Spaces

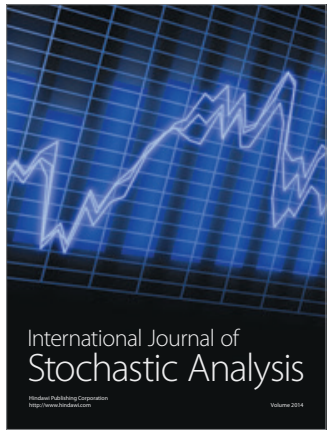

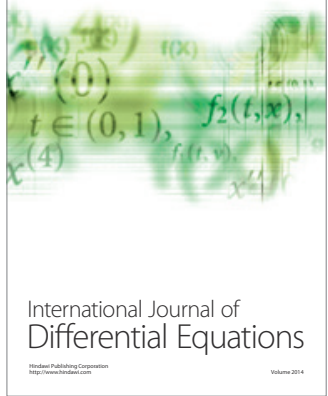
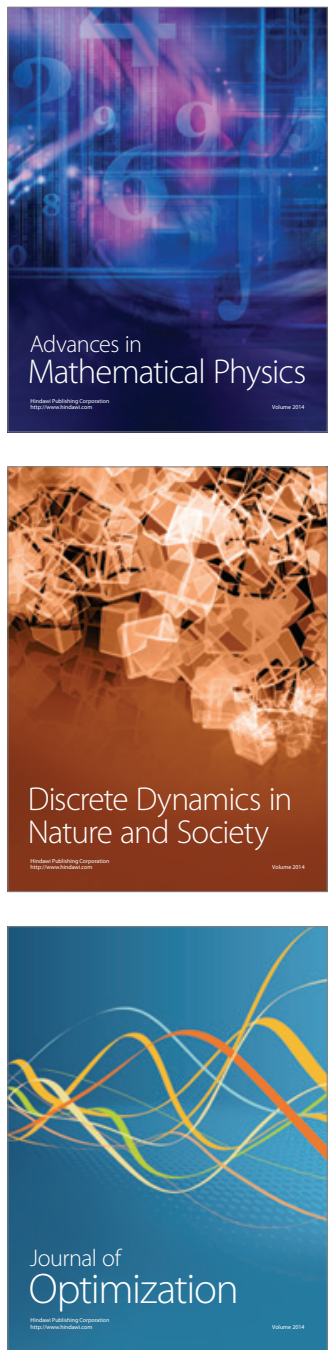